\title{
Importancia de la innovación y su ejecución en la estrategia empresarial
}

\section{Importance of innovation and its execution in the business strategy}

Fernando Andrés Haro Carrillo

Nelson Claudio Córdova Rosas

María Andrea Alvarado Garcés

Universidad Santa María, Ecuador

Autor por correspondencia: fharoc@usm.edu.ec,ncordova777@hotmail.com, malvaradog@usm.edu.ec

Fecha de recepción: 10 de Febrero de 2017 - Fecha de aceptación: 28 de Abril de 2017

Resumen: Uno de los principales puntos de este artículo es el exponer algunos conceptos sobre innovación y clarificar para extraer elementos comunes que nos lleven a desarrollar nuestro propio concepto. Además analizamos los tipos de innovación existente debido a que muchas empresas carecen de criterio para reconocerlas o han mantenido un concepto tan básico de la misma que sólo consideran innovación a la creación de un producto o servicio. Una de las intenciones es desaparecer ese mito. Hacemos referencia a la innovación no sólo en función al área que va a ser mejorada sino a la intensidad en que será planteada. Tenemos claro que innovar es tanto crear algo nuevo, que no existe, como introducir mejoras en procesos ya existentes. Así mismo buscamos responder a las interrogantes ¿Por qué innovan las empresas? ¿Es por estrategia? o ¿Por qué se enfrentan a épocas de cambios o crisis o no tienen más remedio? Una de las respuestas o motivos viene principalmente porque en algún momento de su gestión reciben o buscan información sobre la realidad del entorno económico y comercial de su industria o sector. Es muy fácil encontrar dentro de la planeación estratégica empresarial aspectos como objetivos, estrategias, planes de acción y presupuestos, adema de planes de marketing vinculados a las finanzas internas e incluso planes de desarrollo interno aplicados a los recursos humanos. Pero no es fácil encontrar un plan o estrategia de innovación ya que la mayoría de las organizaciones no lo toma como un eje estratégico o como una herramienta que permitirá el solucionar problemas de crecimiento institucional en el corto, mediano y largo plazo. Por eso mencionamos al liderazgo innovador como clave para transmitir o generar una cultura de innovación dentro de la empresa y que la misma sea considerada estratégica y de igual o mayor importancia que cualquier unidad o departamento. El conseguir esto permitirá a cualquier empresa que haya apostado a combinar la innovación con la estrategia a pensar y valorar qué tipo de innovación quiere y puede poner en marcha.

Palabras claves: innovación; creación; liderazgo innovador

Abstract: One of the main points of this article is to expose some concepts about innovation and clarify them to extract common elements that lead us to develop our own concept. In addition, we analyze the types of innovation that exist because many companies lack the criteria to recognize them or they have maintained such a basic concept of innovation that they only consider it as the creation of a product or service. One of the goals is to disappear that myth. We refer to the innovation not only in function to the area that is going to be improved but to the intensity in which it will be raised. We are clear that innovating is both creating something new, that does not exist, 
as introducing improvements in existing processes. We also seek to answer the questions: Why do companies innovate? Is it because of a strategy? O Why do they faced with times of crisis and have no choice? One of the answers or reasons comes mainly because at some point in their management they receive or look for information on the reality of the economic and commercial environment of their industry or sector. It is very easy to find within strategic business planning aspects such as objectives, strategies, action plans and budgets. Also, marketing plans linked to internal finance and even internal development plans applied to human resources. But it is not easy to find a plan or strategy of innovation since most of organizations do not take it as a strategic axis or as a tool that will solve problems of institutional growth in the short, medium and long term. That is why we mention innovative leadership as a key to transmit or generate a culture of innovation within the company and that it is considered strategic and of equal or greater importance than any unit or department. Achieving this will allow any company that has bet on combining innovation with strategy to think and value what kind of innovation they want and can launch.

Key Words: innovation; creation; innovative leadership

\section{Introducción}

Dentro de la organización y dirección de una empresa existen muchos aspectos a analizar y revisar. Por la experiencia acumulada en estos años consideramos que uno de los factores más importantes o de más alto nivel dentro de las empresas es el estudio de la estrategia empresarial. Este es el punto donde toda la información recopilada en las distintas áreas ya sea primarias o de apoyo converge para transformarse en una decisión que puede ser de corto, mediano o largo plazo y su influencia en el día a día de la empresa se verá paulatinamente reflejado en los estados financieros.

¿Dónde entra la innovación en estos procesos? La innovación no es un concepto nuevo o de este siglo es un tema que se viene discutiendo desde hace más de 70 años y su discusión no está centrada en definición o conceptos sino en cómo diferenciarla, generarla y aplicarla en las diferentes culturas internas de cada empresa, ya que entendemos a cada empresa como un grupo de seres humanos con competencias y características únicas sin dejar de lado al liderazgo gerencial al que cada empresa es sometida como base de la cultura empresarial y es por esto que la innovación como termino no ha sido un problema o el problema, sino el costo que puede involucrar el generarla o el tiempo en que su implementación en la cultura de la organización pueda desarrollarse.

Por esta razón, el objetivo central de este artículo es el dar un punto de vista del por qué la estrategia no puede ni debe ser visualizada como algo aparte de la innovación sino todo lo contrario como un aspecto fundamental en el desarrollo integral de la gestión de toda empresa cualquiera que sea su ramo y el principal objetivo a conseguir. Esto nos llevará a definir la innovación y conocer sus conceptos básicos, los diferentes tipos de innovación y las razones para innovar.

\section{Entendiendo la innovación}

Existen muchas definiciones de innovación de fácil acceso vía web. Expondremos algunos de los conceptos más importantes y de mayor valía para establecer dos conceptos 
centrales en común entre las definiciones: Para el economista austriaco (Schumpeter, 1942), definió a la innovación desde un punto de vista comercial interpretando a la misma como "la introducción de un bien o producto nuevo para los consumidores o de mayor calidad que los anteriores, la introducción de un nuevo método de producción en cualquier sector de una industria, la apertura de nuevos mercados, la mejora en procesos logísticos internos y externos o la mejora estratégica en las formas de competir en una industria llevando a redefinir a la industria"

Dentro de las ideas sobre la innovación más marcadas por Schumpeter, encontramos la de la destrucción creativa, que pone a la innovación como una idea que acaba con las viejas formas de hacer las cosas e introduce nuevos y superiores paradigmas, más productivos y competitivos, eliminando o reduciendo los existentes y desarrollando un constante proceso creativo.

Según (Silverberg \& Soete, 1988), "la innovación debe considerarse como un proceso interactivo en el que la empresa además de adquirir conocimientos mediante su propia experiencia en los procesos de diseño, desarrollo, producción y comercialización, aprende constantemente de sus relaciones con diversas fuentes externas, entre las que podemos anotar a los proveedores, consumidores y diversas instituciones, como las universidades, centros de investigación, consultores y competidores. La interacción entre estos entes difiere según la industria o sector dependiendo de su tecnología, producto o servicio y por sobretodo del entorno y sus tendencias. Por otro lado, (Abernathy \& Clark, 1985), aportan el concepto de transiliencia, que define a la innovación con la capacidad para alterar o destruir los sistemas ya implementados de producción y marketing. Dentro de sus ideas puntualizan que existen innovaciones que pueden dejar fuera de juego a ciertas empresas competidores y otras innovaciones que pueden más bien reforzar el status quo del sector.

Para Peter Drucker, reconocida autoridad en la investigación sobre manejo empresarial, propone que toda organización debe tener a la innovación como su competencia básica y clasifica a la innovación en: mejora, evolución gestionada e innovación. La mejora pretende hacer que todo proceso interno de la empresa sea cada vez más eficiente. La evolución gestionada es el uso de un nuevo producto, proceso o servicio para crear un producto o servicio aún más nuevo y la innovación es el uso sistemático de los cambios en la sociedad, en la economía en la demografía y en la tecnología.

Para finalizar (Mortensen \& Bloch, 2005), explica a la innovación como un concepto de implantación de cambios importantes en el producto, proceso, marketing y organización de la empresa con el firme propósito de mejorar sus resultados. Estos cambios pueden ser realizados mediante la aplicación de nuevos conocimientos adquiridos o desarrollados internamente o externamente mediante servicios de asesoría o compras de tecnología.

Se consideran actividades de innovación a todo proceso de mejora en las áreas científicas, tecnológicas, organizativas, financieras y comerciales. Tanto en las que se haya generado éxito como en las que estén en curso o se encuentre en algún proyecto que por falta de viabilidad no se haya llevado a la práctica. La innovación siempre implica el uso de un conocimiento nuevo o de una combinación de conocimientos ya existentes. 
Desde donde sea que miremos a la innovación la podremos resumir en dos partes bien marcadas: la creación de algo nuevo y su puesta en valor. Y esa novedad puede darse tanto por un descubrimiento científico como por una recombinación de varios conocimientos y tecnologías en uso actual. Por otro lado la puesta en valor se relaciona con la comercialización con éxito y eso quiere decir que para ser considerada innovación debe haber sido aplicada la misma y haber agregado valor comercial a la empresa.

Es importante que establezcamos la diferencia entre un invento y la innovación, el invento normalmente es un evento aleatorio, circunstancial no impulsado por razones económicas ni competitivas, mientras que la innovación tiene como impulso principal al desarrollo comercial del sector o segmento al que nos estamos dirigiendo, esto hace que el estudio del cliente objetivo o la estrategia a implementarse tenga tanto o más peso que la generación de ideas o la gestión de I + D.

Con relación a la I + D + i (Investigación, Desarrollo e Innovación), es de suma importancia dejar claro ciertos puntos. La investigación es un proceso que transforma riqueza en conocimiento mientras que la innovación transforma el conocimiento en riqueza.

\section{Tipos de innovación}

Luego de haber definido varios conceptos sobre lo que es innovación y la importancia en su implementación en el desarrollo empresarial, es importante destacar para romper algunos mitos sobre la innovación principalmente desarrollados entre las pequeñas y medianas empresas, las cuales piensan que no pueden hacer innovación o que solo esta puede ser una aprovechada por las grandes organizaciones. Y llevando más allá la definición de innovación es importante dejar claro que la innovación es una característica de los trabajadores, es decir, proviene o se desarrolla en una cultura corporativa, son habilidades que se tienen, se pueden aprender o desarrollar, porque son las personas, no las empresas, las que innovan.

Citando nuevamente al (Mortensen; et all.), encontramos a la innovación en estos aspectos en las organizaciones:

- Producto/servicio: se genera cuando existe un ingreso en el mercado de nuevos productos o servicios, incluyendo ciertas alteraciones en las especificaciones técnicas, materiales, componentes o en otras características funcionales.

- Proceso: implementación o mejoras en los procesos de logística, fabricación o distribución, que permitan el optimizarlos y ser más productivos.

- Organizacional: Implica un desarrollo en los modelos organizacionales, en la organización del trabajo tanto interna como externa y sus relaciones hacia el exterior.

- Marketing: Son cualquier nuevo método que incluya mejoras significativas tanto en el diseño del producto como en su embalaje, precio, distribución y promoción.

\section{En cuanto a su grado de impacto}

Es importante también destacar que la innovación también se debe medir en cuanto a su grado de impacto o intensidad debido a que esto puede ser parte de una estrategia planificada o 
puede darse por un chispazo (creatividad) que puede generar cambios no solo en la organización sino en la industria y en ciertos casos la economía de un país. Las innovaciones pueden ser:

- Incrementales: Cuando lo que se origina dentro de la empresa son pequeños cambios que generan mejoras en los productos o servicios y que de producirse de forma acumulativa se puede transformar en una ventaja competitiva.

- Radicales: Son mejoras internas o externas que se producen de un momento a otro y que rompen con lo establecido. No pueden ni deben de entenderse como una evolución natural de lo ya existente.

Hay que considerar ciertos aspectos adicionales de la innovación. Primero que no solo es la creación de nuevos productos. Segundo que no es el crear algo de la nada y tercero que para ser considerada innovación debe agregar o generar valor para la organización.

\section{Razones para innovar}

Hay que destacar que el innovar en las organizaciones durante estos últimos años se ha vuelto un tema diferencial y de mucha importancia. Ya que solo las empresas que son capaces de innovar, de reconocer el valor de esa innovación y de ofrecer algo nuevo en el mercado serán consideradas como competitivas y además estarán en condiciones de sobrevivir en su entorno económico actual.

Debido al creciente dinamismo, turbulencia e incertidumbre en el entorno actual se pueden considerar a estos aspectos como reflejo del porque la innovación es tan importante ahora como parte de la gestión.

- Globalización progresiva de mercados

- Ciclo de vida reducida de productos

- Rápidos cambio tecnológicos

- Cambios continuos en patrones de compras de los clientes

Analizando los puntos anteriores convergemos que los mismos exigen una mejora continua de la competitividad y que estas sean de largo plazo o duraderas. Todas las organizaciones deben de asumir a la innovación como un instrumento para la creación de valor en sus productos o servicios, en sus organizaciones y en sus procesos para así mejorar sus capacidades y funcionar con mayor sinergia con su respectivo entorno.

Podemos definir que las principales razones por las que una empresa debe innovar deben de estar alineados con los siguientes razonamientos:

- Los cambios en las empresas no son algo que la innovación ha descubierto sino que han existidos desde siempre.

- La innovación es uno de los enfoques más utilizados por las empresas para canalizar adecuadamente las mejoras generadas en productos, servicios, procesos o marketing. 
- Hay que tomar en consideración no solo el nuevo entorno de los negocios sino el mayor acceso a información actualizada, sería impensable el manejar las organizaciones con sistemas del pasado.

- Es importante que las organizaciones definan y de ser necesario opten por nuevos modelos de gestión de ser el caso en tanto en cuanto los lleve a mejorar su productividad.

\section{Alineando la innovación con la estrategia empresarial}

Para conseguir el alineamiento de la estrategia, la misma deberá ser desarrollada dentro de un marco metodológico que involucra a cuatro factores de suma importancia:

- Misión

- Cultura

- Diagnóstico Inicial

- Análisis del entorno

Además de dos características fundamentales del entorno que representan los factores críticos del desarrollo de la estrategia y la innovación:

- La Globalización

- La Tecnología

Una vez levantados estos puntos se podrá definir los objetivos estratégicos y cuál será la oferta y el segmento a quien buscaremos introducir nuestro producto o servicio. Es exactamente en este punto donde se debe fusionar el alineamiento de la innovación con la estrategia, buscando que la innovación favorezca o impulse a la estrategia.

Entonces la innovación surge como un resultado de la innovación estratégica analizada después de haber revisado la información interna y externa de tres formas:

- Investigación básica: muy relacionada con la ciencia pura (I+D) y no con la puesta en práctica inmediata sino con la profundización del tema de manera bibliográfica.

- Investigación aplicada: Es la parte del I+D donde se enlaza la teoría y el producto. Busca la generación de conocimiento a través de la aplicación de la ciencia básica en problemas ya determinados.

- Investigación tecnológica: Permite a través de la modificación de ciertos aspectos escogidos el desarrollar un producto o servicio con el propósito de implementar una mejora en la productividad de una organización. (inv. Básica + inv. Aplicada)

- Para poder establecer estas etapas que definen procesos de innovación debemos tener claro los siguientes pasos:

- Iniciación del proceso: Existe dentro de la empresa un know how tecnológico en las distintas áreas y el proponerse mejorar ciertos aspectos nos conduce a la innovación ya sea por una necesidad latente o visible o por una situación de mejora continua programada.

- Formulación de idea: Es una etapa de pre diseño donde la técnica y lo económico encuentran su punto medio. La creatividad es fundamental para el desarrollo y deberá ser 
debidamente analizada o costeada para comprometer los recursos necesarios y su planificación en el tiempo.

- Formulación del problema: En ciertas ocasiones la información que permite resolver un problema dentro de la empresa se encuentra a la mano o en su defecto se deberá recurrir a herramienta de investigación y desarrollo. Es una etapa de muchos imprevistos que pueden llevar a la organización a la suspensión temporal o definitiva del proyecto.

- Solución del problema: Esta se puede dar de forma original de un momento a otro o puede ser la adaptación de recursos ya disponibles que nos llevarían a desarrollar una mejora creativa o imitativa.

- Perfeccionamiento o desarrollo: Es la elaboración de prototipos o muestras según las especificaciones estipuladas. Se desarrollan las pruebas y evaluaciones para determinar su factibilidad en la fabricación y posterior puesta en práctica.

- Utilización y difusión: Para que sea considerada innovación debe haber sido introducido o aplicada y debe de mostrar resultados positivos como valor agregado en la empresa.

El ciclo representado en estas etapas comprende desde el producto o servicio, su producción y puesta en el mercado debidamente alineado con la estrategia. No es considerado un proceso de innovación el que no dirija su orientación a conseguir que la organización se vuelva más competitiva en las áreas donde se desarrollarán los proyectos. Pero esta ventaja competitiva lograda existirá en la medida de que la competencia no establezca una estrategia de imitaciónanulación de la diferenciación existente y de las posibilidades que ofrezca el producto de diferenciarse nuevamente.

\section{¿Estrategia innovadora o estrategia de la empresa innovadora?}

Esta combinación es importante destacarla debido a que en los últimos años la tendencia ha sido separar la innovación de la estrategia como actividades divididas de la gestión empresarial y no como un elemento fundamental de la misma.

Según (Drucker, 1985), existen cuatro estrategias específicas innovadoras:

- Meterse a lo grande

- Imitación creativa

- Encontrar y ocupar nichos especializados

- Cambiar las características económicas de un producto, mercado o industria

Considerando a cada una de ellas con sus características diferenciadas pero con la opción de aplicarse simultáneamente ya que no son excluyentes.

A manera de ejemplo podemos citar:

A la empresa Dupont y su propuesta de nylon como "meterse a lo grande". El caso de IBM con Apple en la fabricación del PC como una "imitación creativa". El de Gillette como un caso de "encontrar y ocupar un nicho especializado" y el "cambiar las características económicas de un producto, mercado o industria" a un cambio en el modelo de negocio implantado en la compañía Amazon en su promoción y venta. 
Para esto se necesita de un liderazgo convertido a la innovación el cual hará posible el equilibrio entre la gestión del presente y la del futuro. Pensando en el corto plazo todo podría resultar más o menos estático y estable ya que en el mediano o largo plazo hay una mayor tendencia a que las cosas cambien. Debido a esto podríamos pensar que la implementación de una estrategia innovadora debería ser implementada lo más pronto posible sin descartar los obstáculos propios de una organización y su cultura.

Podemos mencionar que a veces existe una facilidad para generar ideas innovadoras pero no necesariamente tenemos la misma habilidad para volverlas rentables, muchas veces estas ideas son generadas en los mismos ámbitos y no se crean unidades independientes y por último muchas de estas ideas no tienen claro o no mantienen el "Core" o centro del modelo de negocio.

Entonces podemos acotar que para ser innovadores en la estrategia hay que tomar en cuenta muy a fondo los siguientes campos:

- Cultura Organizacional

- Recurso Humano Organizacional

- Estructura Organizacional

- Procesos Organizacionales

Para ser ágiles según las nuevas metodologías y lograr el cambio permanente y continuo de la innovación en la estrategia debemos considerar los cuatro campos antes mencionados aplicando un método dinámico de manera escalonada considerando las siguientes variables:

- La generación de ideas en el arranque de la estrategia

- Buscar separar la nueva unidad de innovación

- Reestructurar los procesos de comunicación interna con la unidad central

- No dejar las discrepancias o tensiones sin atender rápidamente

- Facilitar que la gestión de la nueva unidad sea rápida y efectiva

- Desarrollar una planificación independiente de la nueva unidad con respecto a la central

Está claro que las empresas que buscan generar innovación y la toman como parte de su estrategia son las que buscan adaptarse al entorno y eso les genera casi de manera automática el constantemente innovar su estrategia. No se puede pensar en que ambas son separables pues tienen un mismo fin: Lograr la supervivencia de la organización.

\section{Como ejecutar la innovación}

Para ejecutar la innovación debemos primero asegurarnos que lo que vamos a implementar es realmente es una innovación. ¿Qué elementos deben estar presentes para estar seguros que tenemos una innovación en nuestras manos?

En una primera aproximación a responder esta pregunta diremos que en primer lugar "Innovación es sinónimo de cambio" (Escorsa Castells, 2004), la empresa presenta nuevos esquemas o nuevos enfoques o pone en marcha nuevos procesos de producción, es decir 
claramente cuando hay innovación hay muchos elementos dinámicos en la empresa que van tomando diferentes formas. "la innovación es generar cambios y convertirlos en oportunidades de negocio".

Otro elemento a seguir es que la innovación, siempre persigue un objetivo, que a partir de una necesidad, por ejemplo, se desarrolla un producto, técnica o un servicio útil. Además siempre en innovación está involucrada la palabra "nuevo": aplicada a procesos, productos, enfoques, en cualquier actividad que realice la empresa.

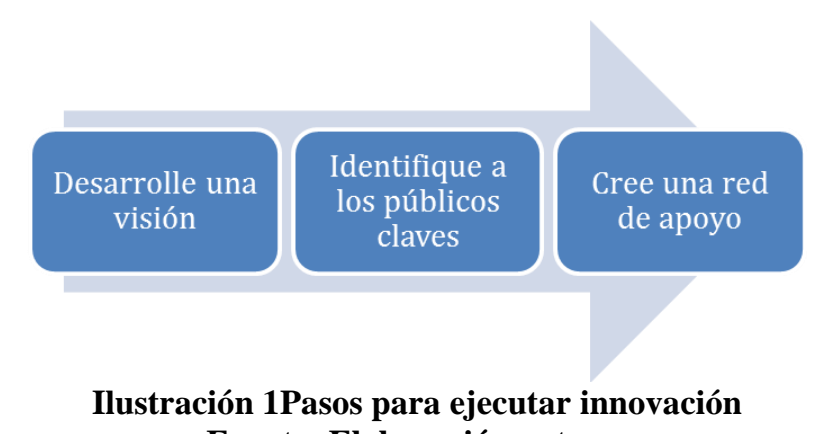

Fuente: Elaboración autores
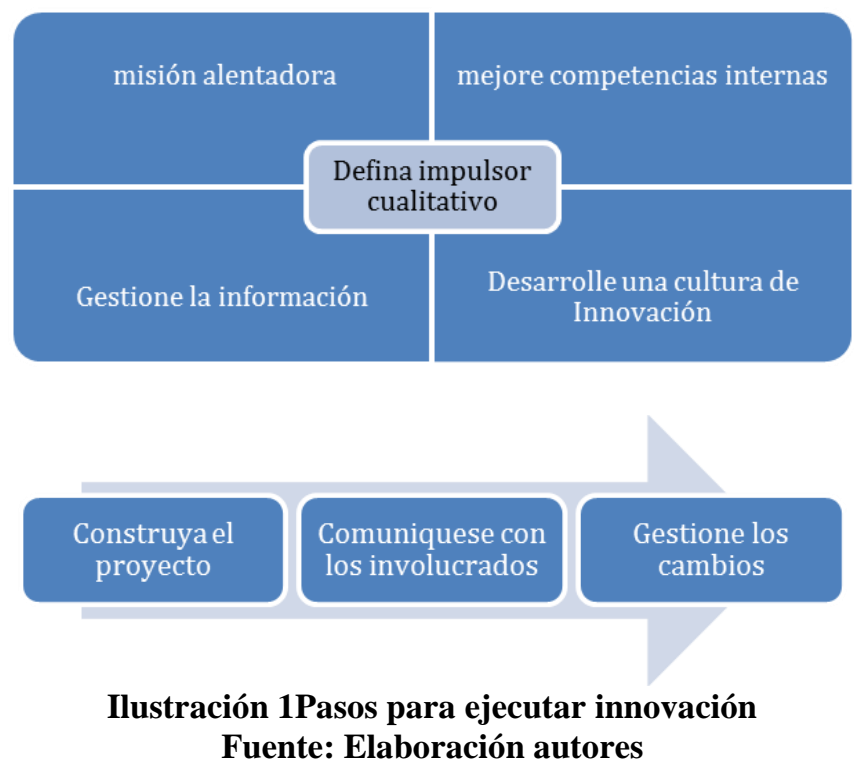

\section{Desarrolle una visión}

Una visión de una empresa es una "imagen" de como se ve la empresa en un futuro, como es su concepción interna con respecto al entorno y a su organización interna. Es una declaración que promueve la empresa a movilizarse hacia un norte al cual mirar y apuntar sus esfuerzos.

Esta visión debe ser compartida por toda la organización y debe ser el credo que lleva cada trabajador en su quehacer diario, por lo que debe ser socializada y entendida por cada uno de los trabajadores sin excepción. 


\section{Identifique los públicos claves}

Los públicos claves son vitales en la proyección de las organizaciones. Son grupos, instituciones u organizaciones las cuales son afectadas directamente por las acciones u omisiones de una empresa, y que además solicitan o reclaman atención, prestación de servicios, recursos, información o resultados de la gestión/administración. La insatisfacción de sus requerimientos o necesidades puede afectar el cumplimiento de la visión establecida que se ha propuesto como empresa.

Entre los más importantes tenemos:

- Accionistas de la organización

- Alta dirección/directorio/junta general

- Clientes actuales

- Clientes potenciales

- Proveedores calificados

- Personal de la organización (talento humano)

- Entidades de control y/o reguladoras

- Comunidad/país en el que desarrolla actividades

- Competencia establecida

\section{Cree una red de apoyo y contactos}

Al construir una red de apoyo que lidere el desarrollo de su idea o estrategia dentro de la organización tendrá un mejor soporte para poder intercambiar inquietudes, discutir mejores soluciones o mejorar la propuesta ya establecida.

Este proceso se inicia con el entusiasmo del generador de esa idea, proceso o estrategia, sin embargo necesitará recursos tanto humanos como financieros para poder desarrollar, implementar y luego evaluar la efectividad de la misma.

Es ahí cuando deberá construir lazos, no sólo de apoyo de colegas, amigos, familiares sino también de personas que tengan un alto impacto en la búsqueda de estos recursos y personas conocedoras de la temática innovadora.

A medida en que las personas se impliquen más en el desarrollo de la propuesta, pedirán una mayor participación en el involucramiento de la misma; es muy común conocer personas que tienen ese proyecto, sin embargo al carecer de estos recursos la red de apoyo que se involucre pedirá en muchos casos hasta un paquete accionario tan representativo que pasará a liderar la toma de decisiones.

Es importante saber escoger cautelosamente su red de apoyo, si bien es cierto la familia y amigos en muchas ocasiones serán quien nos dará ese empujón inicial para no desistir en ese proyecto que a veces es considerado como ambicioso. 
La búsqueda de esa persona que nos generará contactos para el apoyo financiero y de recursos es clave, sin embargo no es la primera a la que se debe acudir.

Si bien hemos hablado de familiares y amigos como primera instancia, aterricemos dentro de una organización. Se necesita ese portavoz que en muchos casos es quién conoce de la temática del proyecto y es a quién respetan mucha dentro de la empresa.

No necesariamente suelen estar en cargos directivos, son personas que gozan de mucha confianza de los dueños de la organización. Su opinión será válida para que un segundo grupo se adhiera a nuestra red de apoyo los que nos darán soporte para diseñar y sobre todo poner en funcionalidad nuestro proyecto, idea o estrategia. Normalmente suelen ser gerentes o jefes que adherentemente al encontrarse en un medio determinado conocen a otros contactos que nos podrán ayudar con información o experticia.

Una vez que el proyecto esté plasmado como una propuesta sostenible y sobretodo confiable para la organización, se deberá recurrir a las altas esferas para conseguir el auspicio del mismo. Es importante no apresurar el paso, pero tampoco abordarlas de forma tardía. Todo se dará a su propio tiempo.

Una vez compactada toda su red de apoyo no olvidar la que podría ser una red de apoyo virtual, deberá evaluar cuando involucrar a sus futuros clientes dentro de la misma.

\section{Desarrolle un impulsor cualitativo}

El concepto es relativamente nuevo y se está usando específicamente en la resolución de problemas en matemáticas, pero su concepto aplica a todo tipo de mejoramiento, creación de valor o resolución de problemas en las empresas.

Un impulsor cualitativo es un "elemento, componente, cualidad o característica perteneciente a la estructura de una empresa o a su contexto, que permite dar un salto cualitativo en la resolución del problema, siendo capaz de proyectar más vías de solución efectivas y claras"1" (Córdova, Sánchez, \& Urrutia, 2016)

Un impulsor cualitativo puede ser un concepto que induzca un cambio de mentalidad en una empresa o sector, de tal manera que provoque cambios tan significativos que impulsan a la empresa hacia un nuevo horizonte que antes no veía y que ahora le promueven beneficios insospechados.

Para encontrarlo es imprescindible poner de manifiesto todas las acciones que realiza la empresa en una pantalla imaginaria y dibujar todas las relaciones e interconexiones de esas acciones realizadas por la empresa para descubrir el elemento clave que me impulsará hacia un nivel superior, este paso es previo al desarrollo de la innovación de cualquier empresa. Este impulsor debe ser tal que relaciona el micro entorno de la empresa con los procesos internos que hace que fluyan los procesos de manera óptima.

\footnotetext{
${ }^{1}$ Definición adaptada para empresas
} 
Ejemplos de impulsores:

\section{Definición de una misión:}

- Microsoft: "poner un computador personal en el escritorio de cada persona", al inicio.

- 3m: "resolver innovadoramente problemas no resueltos".

- Volkswagen (al principio): "fabricar un carro para el pueblo".

- Walt Disney: "hacer feliz a la gente".

- Merck: "preservar y mejorar la vida humana".

- Ronald Reagan: "menos gobierno".

- Gorbachov: "transformemos este sistema. Se salió de su curso por el terror, el absolutismo y la corrupción. Renovémoslo".

- Bell Atlantic: "vamos a ser la compañía líder del mundo en telecomunicaciones y en gerencia de la información".

\section{Valores organizacionales}

La forma para involucrar, para crear y mantener la interrelación social dentro de una organización es en base a un liderazgo positivo en función de los valores organizacionales; revisemos algunos de los enfoques para definirlos:

- La confianza mutua

- El respeto de sí mismo y de los demás

- La empatía

- La flexibilidad

- La preocupación por las necesidades individuales y de grupo

- La participación en la toma de las decisiones

- Las formas de comunicación interna

Ejemplo de valores organizacionales desarrollados y listos para difundir en una organización

- Respeto a las personas

- Excelencia en el servicio

- Trabajo en equipo

- Pasión y diligencia en el trabajo

- Honestidad

\section{Gestione la información}

Desarrolle estrategias de gestión de la información, por ejemplo existen sistemas de Vigilancia tecnológica e inteligencia competitiva que permiten encontrar factores claves para impulsar la innovación en tu empresa. Esta actividad como tal tiene algunos años, pero con la reciente tecnología e internet se hace más fácil de implementar. Es necesario que la empresa 
conozca su entorno, y se mantenga alerta a los cambios: ya sean tecnológicos o nuevos hábitos del mercado, ingreso de nuevos participantes, creación de nuevos mercados, etc.

"Una empresa que tenga acceso a información valiosa para su competitividad, en una forma oportuna y adecuada, que cuente con una cultura en la que promueva la discusión y donde los miembros clave de la empresa mantengan contacto con fuentes externas de in formación y más importante aún, que pueda transformar esta información en un producto inteligente, es decir que brinde resultados de alto valor estratégico, verá incrementada significativamente sus posibilidades para obtener innovaciones exitosas" (Escorsa., 2001)

\section{Desarrolle una cultura de innovación}

La cultura organizacional es la "personalidad" de la empresa; es decir refleja claramente "la manera de como aquí se trabaja”, el “¿cómo se hacen las cosas aquí?”. Es el "corazón y la esencia de la empresa". Son esos patrones, reglas y hábitos "no escritos" que son compartidos por todos y que configuran el modo de actuar de las personas con relación a los públicos claves de la empresa.

Una cultura sólida y sana proporciona los comportamientos deseados en la empresa; le da sentido, dirección, claridad y propósito a la gente, permitiéndoles encauzar sus energías hacia la visión establecida y compartida, participando activamente y de manera emotiva en el cumplimiento de los objetivos trazados.

\section{Construya el proyecto}

En esta etapa se busca desarrollar el proyecto aplicando las herramientas, conocimientos, habilidades y técnicas con el fin de conseguir los objetivos planteados. Esto ha generado algunas metodologías que según su enfoque se sugieren para el desarrollo de proyectos. Las más destacables son:

\section{Gestión de proyectos con enfoque tradicional}

Esta es una metodología muy aplicada en proyectos de construcción o industriales y consisten en dividir el proyecto en procesos que se ejecutan en forma secuencial hasta conseguir el objetivo planteado. Los procesos son:

$\checkmark$ Inicialización: definición inicial del proyecto o fase y la aprobación de su puesta en marcha.

$\checkmark$ Planificación: desarrollo de los planes de gestión según su visión

$\checkmark$ Ejecución: puesta en marcha de lo planeado según su fase o proyecto con el fin de obtener entregables.

$\checkmark$ Seguimiento y control: revisión de lo planeado y ejecutado según la línea base y aplicación de correctivos según las desviaciones encontradas.

$\checkmark$ Cierre: Es la etapa donde culmina el proyecto ya sea porque se han entregado, revisado y aprobado los entregables o porque ya no se va a continuar con el proyecto. En todo caso 
se genera una liberación de los recursos del proyecto y de una retroalimentación de lo aprendido.

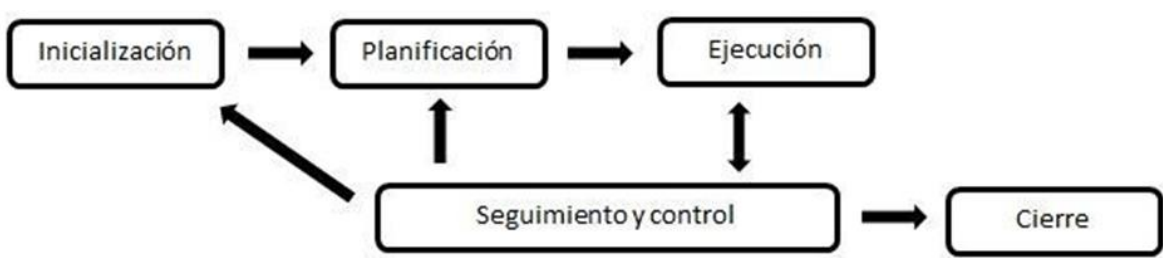

Ilustración 2: Enfoque Tradicional

Fuente: (PMI Institute, 2013)

\section{Gestión de proyectos con Prince2}

A diferencia de los métodos tradicionales, la metodología Prince2 se enfoca más en el producto y sus resultados que en la planificación de las actividades. Y se divide en procesos de la siguiente manera:

$\checkmark$ Puesta en marcha: Descripción, equipo, enfoque y objetivos.

$\checkmark$ Iniciación: Elaboración y aprobación del business case del proyecto o fase.

$\checkmark$ Planificación: Desarrollo de las diferentes áreas del proyecto o fase

$\checkmark$ Dirección: Determinación de las herramientas de control del proyecto, aprobaciones y planificación de las otras etapas.

$\checkmark$ Control de las fases o etapas: Supervisión y presentación de los resultados de cada fase del proyecto.

$\checkmark$ Gestión de las próximas fases: Análisis de lo que debe realizarse al final de cada fase. Asimismo se define las directrices a seguir en el caso de haber o no cumplido con las métricas de gestión.

$\checkmark$ Administración de los entregables: aceptación y aprobación de requisitos de entregables. Incluye contenido, costos, recursos y fechas.

$\checkmark$ Cierre: Asignación formal de actividades de cierre del proyecto a sus responsables y su evaluación.

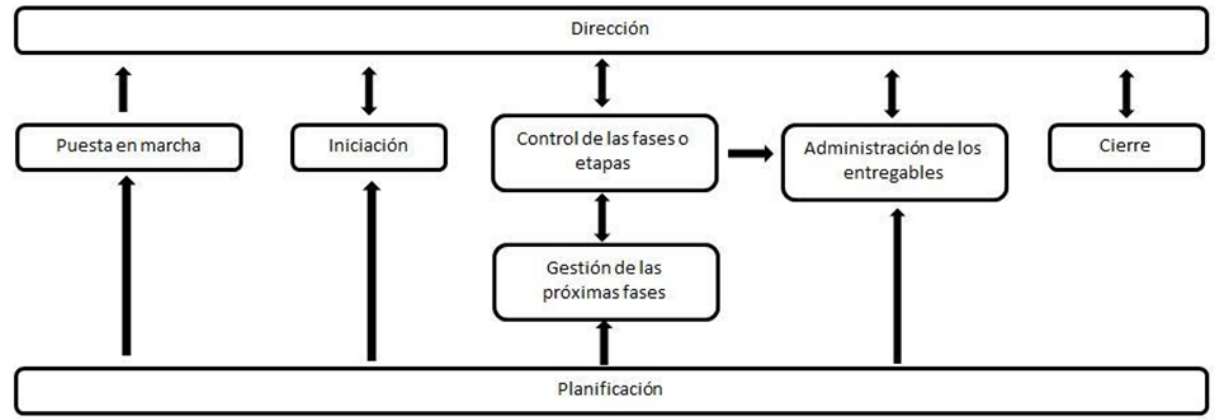

Ilustración 3: Prince 2

Fuente: (CCTA, 2001)

\section{Gestión de proyectos con cadena crítica}


Metodología basada en la teoría de restricciones (TOC), que maximiza el avance del proyecto considerando la incertidumbre y sus limitaciones. Se basa en la aplicación de tres principios:

Identificación de las restricciones del proyecto: Por lo general son el conjunto de tareas que por tiempo o recursos limitan el desarrollo del proceso o etapa. A esto se le llama cadena crítica o camino crítico

Priorización de tareas dentro de la cadena crítica: Los esfuerzos de la dirección del proyecto deben enfocarse en las tareas que sean parte de la cadena crítica, debido a que estas determinarán la finalización del proyecto.

Subordinación del resto de tareas a cadena crítica: Buscar involucrar al resto de tareas y alinearlas con alguna tarea en desarrollo.

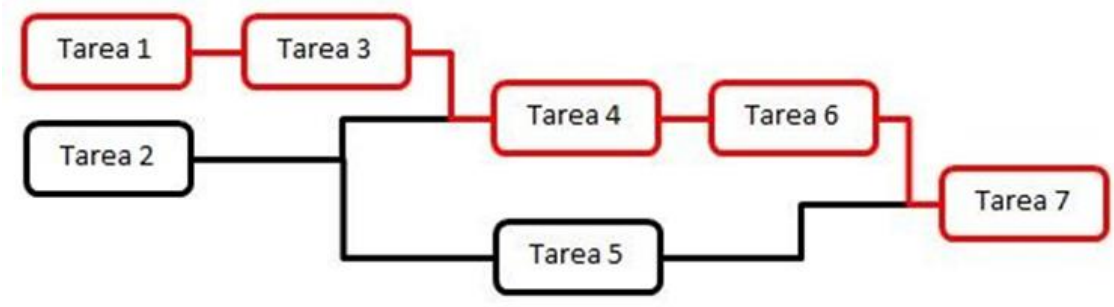

Ilustración 4: Cadena Crítica

Fuente: (PMI Institute, 2013)

\section{Gestión de proyectos AGILE}

Normalmente utilizada en el sector de tecnología debido a su amplia necesidad de flexibilidad y capacidad de modificación a lo largo del proyecto ya que muchas modificaciones se van realizando en el mismo momento de su ejecución. Esta metodología se basa en dividir los proyectos en fases (Sprint) y se estructuran de la siguiente forma:

Inicio: Se escoge del total de objetivos los necesarios para culminar una etapa y desarrollar un producto funcional y a estos se le pone un tiempo determinado de desarrollo, especificando las tareas a seguir.

Desarrollo del Sprint: durante este proceso el equipo va revisando diariamente el cumplimiento de las tareas así como los inconvenientes e impedimentos.

Cierre: Se revisa que se haya cumplido con las tareas y objetivos y exista un producto final, el mismo que al ser revisado por el cliente puede verse inmerso en modificaciones en su objetivo o prioridad. La intención final es lograr un producto que tengas las especificaciones requeridas por el cliente y cumpla con sus expectativas. Esto podría generar una mayor rentabilidad en el producto para la empresa contratante y contratada.

\section{Gestión con los involucrados}


Esta gestión nos lleva a identificar a las personas, grupos u organizaciones que podrían afectarse directamente o ser afectados por las decisiones, actividades o resultados del proceso o proyecto a desarrollarse. Es importante que se identifique para esto la relevancia que tiene cada uno de los interesados en relación a sus intereses, participación, influencia e impacto. Es importante que la comunicación sea fluida y debidamente planificada y organizada para comprender así las necesidades y expectativas que fomenten una adecuada participación de los interesados en las decisiones y actividades del proyecto.

Su satisfacción debe ser considerada como primordial para el correcto desarrollo de la gestión interna y se puede describir su proceso de la siguiente manera:

Identificar a los Interesados: En esta etapa se define quienes son los participantes directos o indirectos del proyecto, la importancia de su intervención y su relevancia en el éxito del proyecto.

Planificar la Gestión de los Interesados: Es importante determinar las estrategias que permitan a los involucrados el desarrollar una gestión eficaz en el proyecto durante su participación en el ciclo de vida del proyecto.

Gestionar la Participación de los Interesados: La comunicación es parte fundamental en esta etapa debido a que se debe trabajar con los interesados de manera que se pueda cubrir cualquier necesidad o incidente de manera rápida y eficaz y así fomentar su participación.

Controlar la Participación de los Interesados: El control de la gestión de los involucrados es parte de la búsqueda del involucramiento de los mismos en los distintos proyectos. Se debe monitorear la relación entre los interesados de manera global y ajustar cualquier estrategia que beneficie su participación.

\section{Gestión de cambios}

Es necesario establecer una gestión eficaz de los cambios que conlleva la innovación establecer hojas de ruta para determinadas áreas no con el objetivo de solamente adaptarse y quedarse en el cambio, sino, estar dispuesto a estar en constante dinamismo de cambios. Es necesario dar ciertos lineamientos, por ejemplo:

$\checkmark \quad$ Movilizar el Cambio a través de un liderazgo Ejecutivo y Participativo.

$\checkmark$ Traducir la innovación en términos operativos en la organización.

$\checkmark$ Alinear la organización con los cambios estratégicos que conlleva la innovación

$\checkmark$ Motivar a los empleados para hacer de la estrategia el trabajo diario de todos.

$\checkmark$ Hacer de la innovación un proceso permanente y continuo.

\section{Conclusiones}

La innovación es un proceso complejo que algunas veces se refleja en un producto o servicio que está a la vanguardia de los tiempos y que muchas veces produce una revolución en la sociedad que es aplicada, otras veces puede ser una nueva forma de hacer las cosas o de interpretarlas, en definitiva concluimos que es algo novedoso, útil aplicable a la sociedad y que 
tiene una durabilidad temporal adecuada que origina nuevos seguidores pero que definitivamente debe de agregar valor al momento de utilizarla tanto para el consumidor como para su creador. Nosotros entendemos entonces como una innovación a "un cambio que da origen a algo nuevo, muy útil para las personas o para el medio ambiente que refleja un resultado de una cadena de pensamientos inteligentes que dan origen a un beneficio perdurable y requerido".

Creemos que para implementar la innovación primero hay que entenderla y socializarla y la empresa en su totalidad debe estar convencida de ella para así proveer de todos los recursos necesarios para su implementación, y nos referimos a recursos económicos, humanos y materiales. Además hacemos hincapié acerca de la necesidad de gestionar la innovación y el encargado de ello es el departamento de I \& D, que investiga y desarrolla nuevas ideas y la mejor utilización de los recursos que hacen que la innovación obtenida permanezca en constante evolución y mejora. (Villegas \& Varela, 2001).

Es necesario definir los pasos para implementar cualquier innovación, puesto que es un proceso complejo que involucra a muchas variables importantes de una empresa, pero la más importante de todas es el talento humano, que si no está involucrado y motivado es muy difícil llevar adelante cualquier innovación, para ello el elemento clave es la comunicación interna y externa incluyendo en una red a los públicos clave o Stakeholders.

La búsqueda de un impulsor cualitativo es necesaria tanto para la motivación interna como para fomentar el trabajo día a día como elemento decisivo para la subsistencia de la innovación. Este impulsor no sólo generaría mejoras internas y externas dentro de la organización sino que la responsabilidad de una empresa que quiere implementar a la innovación dentro de su estrategia está en generar una cultura innovadora que debe ser instaurada de forma que la innovación no sea vista como algo esporádico o de chispazos sino como producto de un proceso organizado, estructurado y sistematizado donde un grupo o unidad dentro de la organización se encargue de manejar la información relacionada del micro y macro entorno para una vez identificados los factores críticos se analicen esas tendencias y estas sean estudiadas para su correcta aplicación dentro de los proyectos futuros establecidos en el plan estratégico.

Pensando y actuando así podremos lograr generar innovaciones más acorde a nuestra realidad económica, financiera, operativa, comercial y cultural y sobretodo dentro de una planeación soportada por una cultura innovadora interna.

\section{Bibliografía}

Abernathy, W. J., \& Clark, K. B. (1985). Innovation: Mapping the winds of creative destruction. Research policy, 3-22.

Anlló, G., \& \& Suárez, Y. D. (2008). Innovación: Algo Más Que I+ D. Evidencias Iberoamericanas A Partir De Las Encuestas De Innovación: Construyendo Las Estrategias Empresarias Competitivas. Innova, 73-103.

Ccta. (2001). Manual Prince 2. Metodología De Gestión De Proyectos. 
Córdova, R., Sánchez, R., \& Urrutia, I. (2016). "Elementos de innovación docente en la mejora del aprendizaje: el impulsor cualitativo y su aplicación en la resolución de problemas en matemáticas”. Revista Ciencias Matemática.ISSN 0256-53.

Drucker, P. F. (1985). The discipline of innovation. Harvard business review, 67-72.

Escorsa Castells, P. \&. (2004). Tecnología e innovación en la empresa (Vol. 148). Catalunya.: Univ. Politèc. De Catalunya.

Escorsa., M. (2001).

García Gálvez, L. X. (2016). La innovación como estrategia de competitividad empresarial. Bachelor's thesis, Universidad Militar Nueva Granada, 1-32.

Isabel Míguez González, M. (2007). Análisis del uso de los conceptos de público, stakeholder y constituent en el marco teórico de las relaciones públicas. Zer. Revista de Estudios de Comunicación, 183-197.

Mortensen, P. S., \& Bloch, C. W. (2005). Oslo Manual-Guidelines for collecting and interpreting innovation data. OECD.

PMI Institute. (2013). Guía de los fundamentos para la dirección de proyectos (Quinta ed.).

Santamaría, L. N. (2009). ¿Hay innovación más allá de la I+ D? El papel de otras actividades innovadoras. Universia Business Review, 102-117.

Schumpeter, J. (1942). Creative destruction. Capitalism, socialism and democracy.

Silverberg, G., \& Soete, L. (1988). Techinal change and economic theory.

Villegas, V., \& Varela, R. (2001). Innovación empresarial: arte y ciencia en la creación de empresas. Pearson educación. 